\title{
STUDY THE EFFECT OF PRE AND POST HARVEST TREATMENTS ON STORABILITY OF SOME VEGETABLES CROPS: \\ 1- EFFECT OF PRE-AND POST HARVEST TREATMENT ON STORABILITY OF POTATO TUBER UNDER AMBIENT TEMPERATURE.
}

\author{
EL-Sayed, Hala A. ; S.T. El-Afifi and Hemat A. EL-Bauome \\ Veget. and Flori. Dept., Fac. Of Agric., Mansoura Univ.
}

\section{ABSTRACT}

Two field experiments, were conducted at a private farm near El-Mansoura Dakahlia Governorate, Egypt during (2006/2007) and (2007/2008) to study the effect of hydro- disillated essential oils (mint, caraway and garlic) salicylic acid compare with hydrogen peroxide and sprout growth inhibitor paclobutrazol $\left(\mathrm{PP}_{333}\right)$ to tubers of spunta cvs on their morphological and biochemical behavior during storage ambient temperature

The obtained results indicated that:-

1- The most superior storage treatments which reduced weight loss, sprouting and damage were garlic oil $(6 \mathrm{ml} / \mathrm{L})$.

2- Tuber dry matter and total soluble solid were significant higher with prolonged of storage period. On the other hand ascorbic acid was significant decreased with prolonged storage period.

3- Dipped tubers with garlic oil (6ml/L) followed by salicylic acid 1000ppm and hydrogen peroxide $1 \%$ significantly reduced higher of total carbohydrate and reducing sugar.

4- Treated tubers with high concentration of essential oil were significantly lower reduced total carbohydrate from the beginning to the end of storage period.

\section{INTRODUCTION}

Potato (Solanum tuberosum L.) is the fourth important crop for human consumption after wheat, maize and rice crops. The importance of potato to agriculture are not only to local consumption, but also to exportation.

A complete controlling of bud development, fully inhibition of sprouting and maintaining certain level of sugars and amino acids are prerequisites and being needed during storage of processing and edible potatoes in refrigerator $\left(10^{\circ} \mathrm{C}\right)$ and Nawalla. Whilst, prolongation of dormancy period, delaying of sprouting and maintaining of bud viability and tuber reserves are being required during storage of potato tuber (Fathy et al., 2005).

Sprouting, weight loss and decay represent serious problems arise during storage of potatoes in Nawalla. But, those known to show no sweetening and sugars rise problems, they fit for processing or edible consumption. On the other hand cold storage $\left(4^{\circ} \mathrm{C}\right)$ of potato seed known to induce serious sweetening and sugars elevation, but it would highly avoid other storage problems. Whereas, it is of high cost and sometimes of insufficient storage capacity. 


\section{E EL-Sayed, Hala A. et al.}

Many chemical compounds (e.g. ethylene, abscisic acid, indole-acetic acid, di-isopropylnaphthalene, CIPC and MH are known to inhibit sprouting. However, most of these substances have never been used commercially because their application can be problematic. Recently, some natural essential oils used and proved efficient sprout suppressive and anti-microbial actions (Knobloch et al., 1986; Vaughn and Spencer, 1991; Oosterhaven et al., 1993 and Hartman et al., 1995). Also, those were effectively improved potato storability, subsequent yield and quality in fall season (El-Awady, 2005).

Elucidation of ambient temperature storage conditions as a high temperature stressful factor induce generation of elevated levels of the toxic and degradable reactive oxygen species (ROS) (Internal injurious oxidative stress) within stressed tissues (Cakmak and Marschner, 1992 and Bowler et al., 1992). The objective of the present study was to determine the effect of. essential oils (mint, caraway and garlic), salicylic acid, hydrogen peroxide and sprout growth inhibitor paclobutrazol ( $\mathrm{PP}_{333}$ ) to tubers of Spunta cvs on their morphological and biochemical behavior during storage at ambient temperature.

\section{MATERIALS AND METHODS}

Two field experiments, were conducted at a private farm near El-Mansoura Dakahlia Governorate, Egypt It aimed to study the effect of hydro distillated essential oils (mint, caraway and garlic), salicylic acid compare with hydrogen peroxide and sprout growth inhibitor paclobutrazol $\left(\mathrm{PP}_{333}\right)$ to tubers of Spunta cvs on their morphological and biochemical behavior during storage at ambient temperature.

planting:- Sprouted Spunta seed tubers were planted on October 20th and 18th in seasons of (2006/2007) and (2007/2008) respectively. The plots area was $10.5 \mathrm{~m}^{2}$ which contained 3 rows, $5 \mathrm{~m}$ length and 0.7 wide. All plants were fertilized with $195 \mathrm{~kg} / \mathrm{fed}$ Ammonium sulfate $(20.6 \mathrm{~N}), 240 \mathrm{~kg} / \mathrm{fed} \mathrm{Ammonium}$ nitrate, $500 \mathrm{~kg} / \mathrm{fed}$ Calcium super phosphate $\left(15.5 \% \mathrm{p}_{2} \mathrm{O} 5\right)$ and $200 \mathrm{~kg} / \mathrm{fed}$ potassium sulfate $\left(48 \% \mathrm{k}_{2} \mathrm{O}\right)$ according to instructions of Ministry of Agriculture. Also all cultural practices such as irrigation, weeds, pests and disease control were added on all plots were done according the instructions of Ministry of Agriculture and were harvested after 105 days from planting dates. Potato tubers of Spunta cvs were saved from the crops of 2007 and 2008 , cured for 15 days, then size graded and those of uniform size (45-60 $\mathrm{mm}$ ) were chosen for storage treatments. Each one included four replicates and all replicate was $12 \mathrm{~kg}$. Storage of potato tuber was carried out for 6 months (after 15 days curing period after harvest) under room condition in case of sackcloth.

\section{The experimental design and treatments:-}

Treatments were arranged in randomized complete block design with 4 replicates. The experiment included two parts :- the first part is spray plants of potato 2 times in the field with emulsion of different essential oils (mint, caraway and garlic) each at 4 and $6 \mathrm{ml} / \mathrm{L}$ at 15 and 30 days before harvest and the second part involved dipping twice with emulsion of different essential oils 
(mint, caraway and garlic) each at 4 and $6 \mathrm{ml} / \mathrm{L}$ salicylic acid 800 and $1000 \mathrm{ppm}$, hydrogen peroxide 0.5 and $1 \%$ and Paclopetrazol $250 \mathrm{ppm}$ during the whole period of storage, the first was at the onset of storage and the second was applied after three month.

The average of normal room temperature and relative humidity \% during the storage months are shown in Table (1).

Table (1): The average of normal room temperature $\left({ }^{\circ} \mathrm{C}\right)$ and relative humidity \% during the two storage seasons of 2007 and 2008.

\begin{tabular}{|c|c|c|c|c|}
\hline \multirow{2}{*}{ months } & \multicolumn{2}{|c|}{ Temperature $\left({ }^{\circ} \mathbf{C}\right)$} & \multicolumn{2}{c|}{ Relative humidity \% } \\
\cline { 2 - 5 } & $\mathbf{2 0 0 7}$ & $\mathbf{2 0 0 8}$ & $\mathbf{2 0 0 7}$ & $\mathbf{2 0 0 8}$ \\
\hline $\mathbf{1}$ & 29 & 29 & 55 & 55 \\
\hline $\mathbf{2}$ & 28 & 29 & 57 & 57 \\
\hline $\mathbf{3}$ & 28 & 30 & 58 & 61 \\
\hline $\mathbf{4}$ & 29 & 32 & 60 & 62 \\
\hline $\mathbf{5}$ & 30 & 32 & 55 & 60 \\
\hline $\mathbf{6}$ & 29 & 31 & 56 & 58 \\
\hline
\end{tabular}

\section{Studied characters}

- Weight loss and sprouting (\%) were determined after two, four and six months of storage

- Damage (\%) were measured after four and six months of storage

- Dry matter content of tuber samples were dried at $70^{\circ} \mathrm{C}$ till constant weight.

- Ascorbic acid (Vitamin C): which was determined using the 2,6 dichlorophenol indophenol method as described by Ranganna (1979).

- Total soluble solids (T.S.S.): were determined using a hand refractometer.

- Chemical analysis of the stored tubers:-

- Total and reducing sugars were extracted according to method of Ranganna (1979) and determined spectrophotometrically at $700 \mathrm{~nm}$ using spekol II (carlzesis) as described by Nelson (1944) with some modifications of Naguib (1964).

- Total carbohydrate content:- was assayed by the phenol-sulphuric acid method (Dubois et al., 1956) which involved extraction with $15 \%$ tri-chloro acetic acid.

- Statistical analysis:-

The obtained data were subjected to analysis of variance (ANOVA) and the treatments means were separated by $\mathrm{F}$-test and the least significant difference (LSD) test at the $5 \%$ level using the statistical package SAS, release 2005 (USA).

\section{RESULTS AND DISCUSSION}

\section{1-Effect of pre and post-harvest treatments on weight loss, sprouting} and damage:-

Data in (Tables 2, 3 and 4) illustrate that all storage treatment significantly suppressed the incidence of weight loss, sprouting percentage and damage as compared with control after four and six months. Also it was 
clear from such data that garlic oil $(6 \mathrm{ml} / \mathrm{L})$ was effectively reduced damage after four and six months after application SA with high concentration during both seasons. Furthermore application of garlic oil $(6 \mathrm{ml} / \mathrm{L})$ reduced weight loss about $23.13 \%$ and $26.2 \%$ in the first and second season respectively

In addition weight loss and sprouting percentage were not significantly influenced by treatment after two months. They explained such effect was explained by Rabbany etal., (1996) who found that most of the essential oils, especially the aldehyde group containing ones seem to inhibit ethylene production by suppressing ACC synthesis. Also, antimicrobial action of essential oils monoterpenes is included. Such results were in accordance with the results of Coleman and Greg (2001); Fathy et al. (2003) and Fathy et al. (2005).

\section{2-Effect of pre and post-harvest treatments on tuber quality:-}

\section{a- Tuber dry matter:-}

Data in (Table 5) show that percentage of tuber dry matter was not significantly influenced by spray essential oil treatment after harvest in both season. Also the same table indicates that tuber dry matter was significantly increased by increasing the storage period. Dipped potato tuber in essential oil at higher concentration show higher dry matter in comparison with those obtained at the beginning of storage period. Barakat (1996) explained that the increament in dry matter percentage could be due to loss of solid substances in potato seed probably higher than water which in turn induced higher dry matter when calculated as percentage.

Table (2): Pre and post-harvest treatments on weight loss (\%) of tubers at different period of storage 2, 4 and 6 months during 2007 and 2008.

\begin{tabular}{|c|c|c|c|c|c|c|}
\hline \multirow{4}{*}{ Treatments } & \multicolumn{6}{|c|}{ Weight loss (\%) } \\
\hline & \multicolumn{6}{|c|}{ Period in months } \\
\hline & \multicolumn{2}{|c|}{2} & \multicolumn{2}{|c|}{4} & \multicolumn{2}{|c|}{6} \\
\hline & 2007 & 2008 & 2007 & 2008 & 2007 & 2008 \\
\hline control & 3.14 & 3.75 & 4.25 & 5.40 & 14.91 & 12.23 \\
\hline Spray plants with mint oil (4 ml/L) & 2.74 & 1.41 & 3.00 & 3.73 & 8.73 & 8.59 \\
\hline Spray plants with mint oil (6 ml/L) & 2.25 & 1.67 & 2.95 & 4.11 & 10.41 & 6.10 \\
\hline Spray plants with caraway oil (4 ml/L) & 1.67 & 0.94 & 2.15 & 3.25 & 9.16 & 9.21 \\
\hline Spray plants with caraway oil (6 ml/L) & 1.9 & 1.04 & 2.25 & 2.67 & 9.14 & 8.27 \\
\hline Spray plants with garlic oil (4 ml/L) & 1.75 & 1.09 & 2.28 & 3.5 & 8.92 & 7.64 \\
\hline Spray plants with garlic oil (6 ml/L) & 2.25 & 0.56 & 2.67 & 3.67 & 9.07 & 8.59 \\
\hline Dipping tubers in mint oil (4 ml/L) & 1.75 & 1.56 & 2.14 & 2.83 & 8.07 & 7.81 \\
\hline Dipping tubers in mint oil (6 ml/L) & 2.42 & 0.73 & 3.21 & 1.67 & 6.58 & 6.08 \\
\hline Dipping tubers in caraway oil (4 ml/L) & 2.94 & 0.68 & 3.13 & 2.33 & 4.96 & 4.85 \\
\hline Dipping tubers in caraway oil (6 ml/L) & 2.25 & 0.76 & 2.86 & 2.0 & 5.08 & 5.38 \\
\hline Dipping tubers in garlic oil (4 ml/L) & 2.75 & 0.48 & 2.14 & 3.0 & 4.81 & 4.67 \\
\hline Dipping tubers in garlic oil (6 ml/L) & 1.50 & 0.54 & 1.98 & 2.5 & 3.42 & 3.34 \\
\hline Dipping tubers in hydrogen peroxide $0.5 \%$ & 1.94 & 1.34 & 1.83 & 2.66 & 3.88 & 4.57 \\
\hline Dipping tubers in hydrogen peroxide 1\% & 1.78 & 1.67 & 1.86 & 3.18 & 3.26 & 4.19 \\
\hline Dipping tubers in salicylic acid 800 ppm & 1.20 & 0.94 & 2.33 & 2.78 & 4.78 & 4.5 \\
\hline Dipping tubers in salicylic acid 1000ppm & 2.18 & 1.28 & 2.56 & 2.69 & 5.50 & 5.00 \\
\hline Dipping tubers in Paclopetrazol 250 ppm & 1.75 & 1.91 & 2.17 & 2.44 & 4.67 & 4.34 \\
\hline L.S.D. at $5 \%$ & NS & NS & 0.93 & 0.76 & 3.67 & 1.49 \\
\hline
\end{tabular}


Table (3): Effect of pre and post-harvest treatments on sprouting behavior of tubers at different period of storage 2, 4 and 6 months during 2007 and 2008.

\begin{tabular}{|c|c|c|c|c|c|c|}
\hline \multirow{4}{*}{ Treatments } & \multirow{2}{*}{\multicolumn{6}{|c|}{$\begin{array}{l}\text { Sprouting percentage } \\
\text { Period in months }\end{array}$}} \\
\hline & & & & & & \\
\hline & \multicolumn{2}{|c|}{2} & \multicolumn{2}{|c|}{4} & \multicolumn{2}{|c|}{6} \\
\hline & 2007 & 2008 & 2007 & 2008 & 2007 & 2008 \\
\hline Control & 0.77 & 0.43 & 14.47 & 13.7 & 20.15 & 20.79 \\
\hline Spray plants with mint oil (4 ml/L) & 0.77 & 0.83 & 10.90 & 11.71 & 17.74 & 18.67 \\
\hline Spray plants with mint oil $(6 \mathrm{ml} / \mathrm{L})$ & 0.89 & 0.33 & 9.73 & 11.54 & 16.64 & 18.65 \\
\hline Spray plants with caraway oil $(4 \mathrm{ml} / \mathrm{L})$ & 0.93 & 0.50 & 11.73 & 14.31 & 16.11 & 17.81 \\
\hline Spray plants with caraway oil (6 ml/L) & 0.17 & 0.80 & 9.31 & 13.40 & 17.99 & 17.72 \\
\hline Spray plants with garlic oil (4 ml/L) & 0.93 & 0.73 & 12.18 & 12.24 & 17.81 & 18.77 \\
\hline Spray plants with garlic oil (6 ml/L) & 0.64 & 0.83 & 11.42 & 11.64 & 19.39 & 18.34 \\
\hline Dipping tubers in mint oil (4 ml/L) & 0.89 & 0.0 & 11.97 & 10.82 & 17.3 & 18.22 \\
\hline Dipping tubers in mint oil (6 ml/L) & 0.0 & 0.0 & 10.04 & 9.91 & 17.01 & 18.79 \\
\hline Dipping tubers in caraway oil (4 ml/L) & 0.0 & 0.0 & 11.11 & 10.79 & 17.22 & 17.71 \\
\hline Dipping tubers in caraway oil $(6 \mathrm{ml} / \mathrm{L})$ & 0.0 & 0.0 & 10.13 & 9.64 & 17.11 & 16.92 \\
\hline Dipping tubers in garlic oil (4 ml/L) & 0.0 & 0.0 & 10.28 & 11.21 & 16.04 & 17.20 \\
\hline Dipping tubers in garlic oil (6 ml/L) & 0.0 & 0.0 & 8.58 & 9.06 & 15.49 & 15.34 \\
\hline Dipping tubers in hydrogen peroxide $0.5 \%$ & 0.0 & 0.0 & 10.46 & 10.68 & 17.92 & 17.12 \\
\hline Dipping tubers in hydrogen peroxide $1 \%$ & 0.0 & 0.0 & 9.73 & 8.36 & 18.66 & 18.28 \\
\hline Dipping tubers in salicylic acid 800 ppm & 0.0 & 0.0 & 9.01 & 9.43 & 17.77 & 17.21 \\
\hline $\begin{array}{l}\text { Dipping tubers in salicylic acid } 1000 \\
\text { 1000ppm }\end{array}$ & 0.0 & 0.0 & 10.24 & 10.48 & 17.76 & 18.62 \\
\hline Dipping tubers in Paclopetrazol 250 ppm & 0.0 & 0.0 & 7.49 & 8.48 & 16.93 & 18.01 \\
\hline L.S.D. at $5 \%$ & Ns & Ns & 6.32 & 5.11 & 1.48 & 2.03 \\
\hline
\end{tabular}

Table (4): Effect of pre and post-harvest treatments on damage percentage of tubers at different period storage 4 and 6 months during 2007 and 2008.

\begin{tabular}{|c|c|c|c|c|}
\hline \multirow{4}{*}{ Treatments } & \multicolumn{4}{|c|}{ damage percentage (\%) } \\
\hline & \multicolumn{4}{|c|}{ Period in months } \\
\hline & \multicolumn{2}{|c|}{4} & \multicolumn{2}{|c|}{6} \\
\hline & 2007 & 2008 & 2007 & 2008 \\
\hline control & 6.41 & 4.21 & 9.45 & 10.44 \\
\hline Spray plants with mint oil (4 ml/L) & 3.33 & 3.67 & 10.66 & 7.59 \\
\hline Spray plants with mint oil $(6 \mathrm{ml} / \mathrm{L})$ & 3.33 & 3.33 & 9.01 & 8.98 \\
\hline Spray plants with caraway oil ( $4 \mathrm{ml} / \mathrm{L})$ & 4.00 & 4.00 & 8.11 & 8.12 \\
\hline Spray plants with caraway oil ( $6 \mathrm{ml} / \mathrm{L})$ & 3.56 & 3.45 & 9.46 & 8.44 \\
\hline Spray plants with garlic oil $(4 \mathrm{ml} / \mathrm{L})$ & 1.98 & 3.61 & 11.91 & 7.93 \\
\hline Spray plants with garlic oil (6 ml/L) & 2.15 & 3.67 & 9.82 & 8.75 \\
\hline Dipping tubers in mint oil (4 ml/L) & 3.41 & 3.58 & 9.91 & 7.68 \\
\hline Dipping tubers in mint oil (6 ml/L) & 3.67 & 1.25 & 9.00 & 7.23 \\
\hline Dipping tubers in caraway oil (4 ml/L) & 3.45 & 3.21 & 6.21 & 8.92 \\
\hline Dipping tubers in caraway oil (6 ml/L) & 3.89 & 2.22 & 6.86 & 6.44 \\
\hline Dipping tubers in garlic oil (4 ml/L) & 2.18 & 3.26 & 7.49 & 7.40 \\
\hline Dipping tubers in garlic oil (6 ml/L) & 2.89 & 2.22 & 5.63 & 5.76 \\
\hline Dipping tubers in hydrogen peroxide $0.5 \%$ & 3.22 & 1.78 & 6.31 & 6.80 \\
\hline Dipping tubers in hydrogen peroxide $1 \%$ & 3.53 & 2.54 & 6.00 & 6.50 \\
\hline Dipping tubers in salicylic acid 800 ppm & 3.25 & 5.48 & 9.9 & 7.83 \\
\hline Dipping tubers in salicylic acid 1000ppm & 3.05 & 3.29 & 5.89 & 6.04 \\
\hline Dipping tubers in Paclopetrazol 250 ppm & 4.03 & 3.48 & 8.66 & 8.72 \\
\hline L.S.D. at $5 \%$ & 2.04 & 1.81 & 2.08 & 1.53 \\
\hline
\end{tabular}


E EL-Sayed, Hala A. et al.

Table (5): Effect of pre and post-harvest treatments on tuber dry matter (\%) at the beginning of storage and at different period of storage 2, 4 and 6 months during 2007 and 2008.

\begin{tabular}{|c|c|c|c|c|c|c|c|c|}
\hline \multirow{4}{*}{ Treatments } & \multicolumn{8}{|c|}{ Tuber dry matter (\%) } \\
\hline & \multirow{2}{*}{\multicolumn{2}{|c|}{0 time }} & \multicolumn{6}{|c|}{ Period in months } \\
\hline & & & \multicolumn{2}{|c|}{2} & \multicolumn{2}{|c|}{4} & \multicolumn{2}{|c|}{6} \\
\hline & 2007 & 2008 & 2007 & 2008 & 2007 & 2008 & 2007 & 2008 \\
\hline control & 17.63 & 18.65 & 17.81 & 18.83 & 17.76 & 19.65 & 19.88 & 20.02 \\
\hline $\begin{array}{l}\text { Spray plants with mint oil (4 } \\
\text { ml/L) }\end{array}$ & 18.14 & 18.40 & 18.91 & 19.24 & 19.90 & 19.70 & 20.69 & 20.40 \\
\hline $\begin{array}{l}\text { Spray plants with mint oil (6 } \\
\text { ml/L) }\end{array}$ & 17.43 & 17.93 & 18.08 & 18.47 & 18.85 & 19.18 & 19.77 & 20.50 \\
\hline $\begin{array}{l}\text { Spray plants with caraway oil } \\
(4 \mathrm{ml} / \mathrm{L})\end{array}$ & 18.23 & 18.72 & 18.77 & 18.85 & 18.59 & 19.23 & 20.46 & 19.81 \\
\hline $\begin{array}{l}\text { Spray plants with caraway oil } \\
(6 \mathrm{ml} / \mathrm{L})\end{array}$ & 18.05 & 18.23 & 18.26 & 19.15 & 19.44 & 19.26 & 20.95 & 20.33 \\
\hline $\begin{array}{l}\text { Spray plants with garlic oil (4 } \\
\mathrm{ml} / \mathrm{L})\end{array}$ & 17.84 & 16.49 & 18.85 & 18.20 & 19.09 & 19.20 & 20.74 & 20.12 \\
\hline $\begin{array}{l}\text { Spray plants with garlic oil (6 } \\
\mathrm{ml} / \mathrm{L})\end{array}$ & 18.29 & 19.36 & 18.33 & 20.04 & 19.02 & 19.20 & 20.74 & 20.12 \\
\hline $\begin{array}{l}\text { Dipping tubers in mint oil (4 } \\
\text { ml/L) }\end{array}$ & 17.63 & 18.65 & 18.90 & 18.33 & 19.70 & 18.84 & 20.50 & 19.64 \\
\hline $\begin{array}{l}\text { Dipping tubers in mint oil (6 } \\
\text { ml/L) }\end{array}$ & 17.63 & 18.65 & 18.12 & 18.89 & 18.18 & 20.25 & 21.00 & 21.57 \\
\hline $\begin{array}{l}\text { Dipping tubers in caraway oil } \\
(4 \mathrm{ml} / \mathrm{L})\end{array}$ & 17.63 & 18.65 & 18.60 & 19.26 & 20.21 & 19.64 & 20.51 & 21.02 \\
\hline $\begin{array}{l}\text { Dipping tubers in caraway oil } \\
(6 \mathrm{ml} / \mathrm{L})\end{array}$ & 17.63 & 18.65 & 18.64 & 19.86 & 19.02 & 20.20 & 20.61 & 21.29 \\
\hline $\begin{array}{l}\text { Dipping tubers in garlic oil (4 } \\
\mathrm{ml} / \mathrm{L})\end{array}$ & 17.63 & 18.65 & 18.03 & 19.72 & 19.81 & 20.26 & 20.74 & 20.19 \\
\hline $\begin{array}{l}\text { Dipping tubers in garlic oil (6 } \\
\mathrm{ml} / \mathrm{L})\end{array}$ & 17.63 & 18.65 & 17.94 & 19.68 & 18.00 & 20.25 & 21.81 & 21.34 \\
\hline $\begin{array}{l}\text { Dipping tubers in hydrogen } \\
\text { peroxide } 5 \% 0.5 \%\end{array}$ & 17.63 & 18.65 & 18.89 & 17.76 & 18.17 & 18.17 & 20.05 & 19.49 \\
\hline $\begin{array}{l}\text { Dipping tubers in hydrogen } \\
\text { peroxide } 1 \%\end{array}$ & 17.63 & 18.65 & 18.31 & 18.80 & 18.99 & 18.97 & 20.00 & 20.00 \\
\hline $\begin{array}{l}\text { Dipping tubers in salicylic } \\
\text { acid } 800 \text { ppm }\end{array}$ & 17.63 & 18.65 & 18.00 & 18.00 & 18.67 & 19.21 & 20.00 & 19.97 \\
\hline $\begin{array}{l}\text { Dipping tubers in salicylic } \\
\text { acid 1000ppm }\end{array}$ & 17.63 & 18.65 & 18.40 & 18.20 & 19.46 & 19.31 & 20.67 & 20.15 \\
\hline $\begin{array}{l}\text { Dipping tubers in } \\
\text { Paclopetrazol } 250 \text { ppm }\end{array}$ & 17.63 & 18.65 & 18.33 & 18.53 & 19.28 & 19.23 & 20.97 & 20.29 \\
\hline L.S.D. at $5 \%$ & NS & NS & NS & 0.75 & 1.02 & 0.71 & 1.35 & 1.34 \\
\hline
\end{tabular}

b- Ascorbic acid:-

Ascorbic acid in tuber is present in Table (6) It shows that all treatments had significant effect on Ascorbic acid after four and six months. There is decrease in Ascorbic acid from the beginning to the end of storage period meanwhile, dipped in garlic oil $(6 \mathrm{ml} / \mathrm{l})$ and salicylic acid $1000 \mathrm{ppm}$ were high effective in protection of potato tubers against the oxidative ascorbic acid. Similar result was obtained by Fathy et al. (2005). 
Table (6): Effect of pre and post-harvest treatments on Ascorbic acid (Vitamin C) content of tubers at the beginning of storage and at different period of storage 2, 4 and 6 months during 2007 and 2008.

\begin{tabular}{|c|c|c|c|c|c|c|c|c|}
\hline \multirow{4}{*}{ Treatments } & \multicolumn{8}{|c|}{ Ascorbic acid (Vitamin C) $\mathbf{m g} / 100 \mathrm{~g}$ fresh wt. } \\
\hline & \multirow{2}{*}{\multicolumn{2}{|c|}{0 time }} & \multicolumn{6}{|c|}{ Period in months } \\
\hline & & & \multicolumn{2}{|c|}{2} & \multicolumn{2}{|c|}{4} & \multicolumn{2}{|c|}{6} \\
\hline & 2007 & 2008 & 2007 & 2008 & 2007 & 2008 & 2007 & 2008 \\
\hline Control & 20.51 & 20.73 & 19.42 & 17.59 & 17.59 & 17.17 & 13.88 & 13.47 \\
\hline $\begin{array}{l}\text { Spray plants with mint oil (4 } \\
\text { ml/L) }\end{array}$ & 21.15 & 20.64 & 19.47 & 19.69 & 17.92 & 17.68 & 16.30 & 16.45 \\
\hline $\begin{array}{l}\text { Spray plants with mint oil (6 } \\
\text { ml/L) }\end{array}$ & 20.96 & 22.22 & 20.37 & 20.11 & 17.85 & 18.39 & 13.88 & 14.83 \\
\hline $\begin{array}{l}\text { Spray plants with caraway oil } \\
(4 \mathrm{ml} / \mathrm{L})\end{array}$ & 23.85 & 22.18 & 18.50 & 19.35 & 16.67 & 17.68 & 14.22 & 14.81 \\
\hline $\begin{array}{l}\text { Spray plants with caraway oil } \\
(6 \mathrm{ml} / \mathrm{L})\end{array}$ & 23.89 & 16.05 & 18.55 & 18.60 & 17.37 & 17.49 & 15.41 & 15.81 \\
\hline $\begin{array}{l}\text { Spray plants with garlic oil (4 } \\
\mathrm{ml} / \mathrm{L})\end{array}$ & 22.25 & 22.26 & 18.66 & 19.44 & 18.56 & 17.17 & 14.00 & 15.19 \\
\hline $\begin{array}{l}\text { Spray plants with garlic oil (6 } \\
\text { ml/L) }\end{array}$ & 20.41 & 19.47 & 19.74 & 21.05 & 16.7 & 17.68 & 12.17 & 12.63 \\
\hline $\begin{array}{l}\text { Dipping tubers in mint oil (4 } \\
\text { ml/L) }\end{array}$ & 20.51 & 20.73 & 18.96 & 20.45 & 16.67 & 17.78 & 16.16 & 15.14 \\
\hline $\begin{array}{l}\text { Dipping tubers in mint oil (6 } \\
\text { ml/L) }\end{array}$ & 20.51 & 20.73 & 18.32 & 19.44 & 16.68 & 17.99 & 15.74 & 14.5 \\
\hline $\begin{array}{l}\text { Dipping tubers in caraway oil } \\
(4 \mathrm{ml} / \mathrm{L})\end{array}$ & 20.51 & 20.73 & 20.21 & 19.78 & 17.61 & 17.59 & 13.93 & 15.91 \\
\hline $\begin{array}{l}\text { Dipping tubers in caraway oil } \\
(6 \mathrm{ml} / \mathrm{L})\end{array}$ & 20.51 & 20.73 & 20.89 & 19.70 & 17.59 & 17.24 & 16.93 & 16.67 \\
\hline $\begin{array}{l}\text { Dipping tubers in garlic oil (4 } \\
\mathrm{ml} / \mathrm{L})\end{array}$ & 20.51 & 20.73 & 20.91 & 19.78 & 19.61 & 18.52 & 17.59 & 14.39 \\
\hline $\begin{array}{l}\text { Dipping tubers in garlic oil (6 } \\
\mathrm{ml} / \mathrm{L})\end{array}$ & 20.51 & 20.73 & 20.83 & 19.36 & 19.37 & 17.51 & 17.69 & 17.35 \\
\hline $\begin{array}{l}\text { Dipping tubers in hydrogen } \\
\text { peroxide } 0.5 \%\end{array}$ & 20.51 & 20.73 & 20.45 & 19.69 & 19.32 & 19.02 & 16.29 & 16.16 \\
\hline $\begin{array}{l}\text { Dipping tubers in hydrogen } \\
\text { peroxide } 1 \%\end{array}$ & 20.51 & 20.73 & 19.95 & 20.11 & 17.42 & 18.35 & 14.39 & 13.64 \\
\hline $\begin{array}{l}\text { Dipping tubers in salicylic acid } \\
800 \text { ppm }\end{array}$ & 20.51 & 20.73 & 18.18 & 18.60 & 17.67 & 17.68 & 17.50 & 16.67 \\
\hline $\begin{array}{l}\text { Dipping tubers in salicylic acid } \\
1000 \text { ppm }\end{array}$ & 20.51 & 20.73 & 19.32 & 19.02 & 20.51 & 19.37 & 17.55 & 16.80 \\
\hline $\begin{array}{l}\text { Dipping tubers in } \\
\text { Paclopetrazol } 250 \text { ppm }\end{array}$ & 20.51 & 20.73 & 18.18 & 18.60 & 18.18 & 18.18 & 17.05 & 16.42 \\
\hline L.S.D. at $5 \%$ & NS & NS & NS & 1.72 & 2.64 & 2.80 & 1.98 & 2.12 \\
\hline
\end{tabular}

\section{c- Total soluble solid:-}

It was obvious from Table (7) that T.S.S increased gradually with increasing storage period. The results also indicated that application of garlic oil $(6 \mathrm{ml} / \mathrm{l})$ and caraway oil $(6 \mathrm{ml} / \mathrm{l})$ significantly increased T.S.S at the end of storage period. 
Table (7): Effect of pre and post-harvest treatments on Total soluble solids (T.S.S.) of tubers at the beginning of storage and at different period of storage 2, 4 and 6 months during 2007 and 2008.

\begin{tabular}{|c|c|c|c|c|c|c|c|c|}
\hline \multirow{4}{*}{ Treatments } & \multicolumn{8}{|c|}{ TSS \% } \\
\hline & \multirow{2}{*}{\multicolumn{2}{|c|}{0 time }} & \multicolumn{6}{|c|}{ Period in months } \\
\hline & & & \multicolumn{2}{|c|}{2} & \multicolumn{2}{|c|}{4} & \multicolumn{2}{|c|}{6} \\
\hline & 2007 & 2008 & 2007 & 2008 & 2007 & 2008 & 2007 & 2008 \\
\hline control & 4.93 & 4.93 & 5.20 & 5.20 & 5.33 & 5.33 & 5.73 & 6.17 \\
\hline $\begin{array}{l}\text { Spray plants with mint oil (4 } \\
\mathrm{ml} / \mathrm{L})\end{array}$ & 4.93 & 4.90 & 5.00 & 5.67 & 5.07 & 6.17 & 6.86 & 6.50 \\
\hline $\begin{array}{l}\text { Spray plants with mint oil (6 } \\
\mathrm{ml} / \mathrm{L})\end{array}$ & 5.00 & 4.75 & 5.00 & 5.33 & 5.20 & 5.70 & 6.8 & 6.33 \\
\hline $\begin{array}{l}\text { Spray plants with caraway oil } \\
(4 \mathrm{ml} / \mathrm{L})\end{array}$ & 4.07 & 4.90 & 5.07 & 5.03 & 5.87 & 5.83 & 7.53 & 6.17 \\
\hline $\begin{array}{l}\text { Spray plants with caraway oil } \\
(6 \mathrm{ml} / \mathrm{L})\end{array}$ & 4.00 & 4.00 & 5.00 & 5.27 & 4.07 & 5.40 & 5.53 & 7.17 \\
\hline $\begin{array}{l}\text { Spray plants with garlic oil (4 } \\
\text { ml/L) }\end{array}$ & 4.83 & 4.65 & 4.93 & 5.27 & 5.07 & 4.50 & 6.67 & 6.83 \\
\hline $\begin{array}{l}\text { Spray plants with garlic oil (6 } \\
\mathrm{ml} / \mathrm{L} \text { ) }\end{array}$ & 5.10 & 5.15 & 5.33 & 5.10 & 6 & 5.67 & 6.67 & 6.50 \\
\hline $\begin{array}{l}\text { Dipping tubers in mint oil (4 } \\
\mathrm{ml} / \mathrm{L})\end{array}$ & 4.93 & 4.93 & 4.97 & 5.50 & 5.47 & 5.13 & 6.63 & 5.83 \\
\hline \begin{tabular}{|l|}
$\begin{array}{l}\text { Dipping tubers in mint oil (6 } \\
\mathrm{ml} / \mathrm{L})\end{array}$ \\
\end{tabular} & 4.93 & 4.93 & 5.00 & 5.00 & 5.3 & 5.00 & 6.7 & 6.33 \\
\hline $\begin{array}{l}\text { Dipping tubers in caraway oil } \\
(4 \mathrm{ml} / \mathrm{L})\end{array}$ & 4.93 & 4.93 & 5.13 & 5.10 & 5.4 & 4.90 & 6.83 & 7.33 \\
\hline $\begin{array}{l}\text { Dipping tubers in caraway oil } \\
(6 \mathrm{ml} / \mathrm{L})\end{array}$ & 4.93 & 4.93 & 4.87 & 5.60 & 5.2 & 5.80 & 6.57 & 7.17 \\
\hline $\begin{array}{l}\text { Dipping tubers in garlic oil (4 } \\
\mathrm{ml} / \mathrm{L})\end{array}$ & 4.93 & 4.93 & 4.73 & 5.00 & 5.07 & 5.13 & 7.13 & 7.50 \\
\hline $\begin{array}{l}\text { Dipping tubers in garlic oil (6 } \\
\mathrm{ml} / \mathrm{L})\end{array}$ & 4.93 & 4.93 & 4.73 & 5.00 & 5.27 & 5.13 & 6.90 & 7.17 \\
\hline $\begin{array}{l}\text { Dipping tubers in hydrogen } \\
\text { peroxide } 0.5 \% \% 0.5 \%\end{array}$ & 4.93 & 4.93 & 4.90 & 4.60 & 5.17 & 5.17 & 6.50 & 6.33 \\
\hline $\begin{array}{l}\text { Dipping tubers in hydrogen } \\
\text { peroxide } 1 \%\end{array}$ & 4.93 & 4.93 & 5.00 & 4.27 & 5.77 & 5.67 & 6.07 & 6.17 \\
\hline $\begin{array}{l}\text { Dipping tubers in salicylic } \\
\text { acid } 800 \text { ppm }\end{array}$ & 4.93 & 4.93 & 5.60 & 4.93 & 5.67 & 5.93 & 6.33 & 6.17 \\
\hline $\begin{array}{l}\text { Dipping tubers in salicylic } \\
\text { acid 1000ppm }\end{array}$ & 4.93 & 4.93 & 5.33 & 5.00 & 5.83 & 5.67 & 6.33 & 6.33 \\
\hline \begin{tabular}{|l} 
Dipping tubers in \\
Paclopetrazol 250 ppm
\end{tabular} & 4.93 & 4.93 & 5.50 & 4.43 & 5.83 & 5.67 & 6.67 & 6.23 \\
\hline L.S.D. at $5 \%$ & NS & NS & 0.37 & 0.81 & 0.41 & 0.81 & 0.62 & 0.71 \\
\hline
\end{tabular}

\section{3- Chemical composition of tuber:-}

\section{a- Total and reducing sugars:-}

Data in Table (8 and 9) indicate that total and reducing sugars were significantly increased by increasing the storage period. Whereas, spray plants with essential oil had not significantly affect on studied characters at the beginning of storage. In addition total and reducing sugars slowly increased when tubers dipped in garlic oil $(6 \mathrm{ml} / \mathrm{l})$ followed by salicylic acid $1000 \mathrm{ppm}$ and hydrogen peroxide 1\%. This reflected and confirmed their potent suppressive effect on the enzymatic changes of sugars (Vander Plas, 1987; Davies, 1990; El-Wady et al., 2002 and Fathy et al., 2005). 
J. Agric. Sci. Mansoura Univ., 34 (10), October, 2009

Table (8): Effect of pre and post-harvest treatments on total sugar $(\mathrm{mg} / 100 \mathrm{~g})$ at the beginning of storage and at different period of storage 2, 4 and 6 months during 2007 and 2008.

\begin{tabular}{|c|c|c|c|c|c|c|c|c|}
\hline \multirow{4}{*}{ Treatments } & \multicolumn{8}{|c|}{ Total sugar } \\
\hline & \multirow{2}{*}{\multicolumn{2}{|c|}{0 time }} & \multicolumn{6}{|c|}{ Period in months } \\
\hline & & & \multicolumn{2}{|c|}{2} & \multicolumn{2}{|c|}{4} & \multicolumn{2}{|c|}{6} \\
\hline & 2007 & 2008 & 2007 & 2008 & 2007 & 2008 & 2007 & 2008 \\
\hline control & 1.75 & 1.69 & 1.79 & 2.10 & 2.03 & 2.06 & 2.30 & 2.56 \\
\hline $\begin{array}{l}\text { Spray plants with mint oil (4 } \\
\text { ml/L) }\end{array}$ & 1.63 & 1.73 & 1.84 & 1.79 & 1.81 & 1.81 & 2.14 & 2.15 \\
\hline $\begin{array}{l}\text { Spray plants with mint oil (6 } \\
\mathrm{ml} / \mathrm{L})\end{array}$ & 1.74 & 1.12 & 1.82 & 1.63 & 1.93 & 1.96 & 2.13 & 2.14 \\
\hline $\begin{array}{l}\text { Spray plants with caraway oil } \\
(4 \mathrm{ml} / \mathrm{L})\end{array}$ & 1.83 & 1.51 & 2.08 & 1.74 & 2.31 & 1.91 & 2.35 & 2.20 \\
\hline $\begin{array}{l}\text { Spray plants with caraway oil } \\
(6 \mathrm{ml} / \mathrm{L})\end{array}$ & 1.70 & 1.37 & 1.89 & 1.55 & 1.95 & 1.97 & 2.29 & 2.09 \\
\hline $\begin{array}{l}\text { Spray plants with garlic oil (4 } \\
\mathrm{ml} / \mathrm{L})\end{array}$ & 1.43 & 1.48 & 1.98 & 1.49 & 1.92 & 1.83 & 2.21 & 1.98 \\
\hline $\begin{array}{l}\text { Spray plants with garlic oil (6 } \\
\mathrm{ml} / \mathrm{L})\end{array}$ & 1.73 & 1.75 & 1.94 & 1.91 & 2.17 & 1.80 & 2.06 & 2.07 \\
\hline \begin{tabular}{|l} 
Dipping tubers in mint oil (4 \\
$\mathrm{ml} / \mathrm{L})$
\end{tabular} & 1.75 & 1.75 & 1.64 & 1.84 & 1.86 & 1.67 & 1.91 & 2.44 \\
\hline $\begin{array}{l}\text { Dipping tubers in mint oil (6 } \\
\mathrm{ml} / \mathrm{L})\end{array}$ & 1.75 & 1.75 & 1.79 & 1.70 & 2.19 & 1.81 & 2.31 & 2.70 \\
\hline $\begin{array}{l}\text { Dipping tubers in caraway oil } \\
(4 \mathrm{ml} / \mathrm{L})\end{array}$ & 1.75 & 1.69 & 1.12 & 1.96 & 1.86 & 1.44 & 2.16 & 1.86 \\
\hline $\begin{array}{l}\text { Dipping tubers in caraway oil } \\
(6 \mathrm{ml} / \mathrm{L})\end{array}$ & 1.75 & 1.69 & 1.77 & 1.71 & 1.82 & 1.14 & 2.07 & 1.95 \\
\hline $\begin{array}{l}\text { Dipping tubers in garlic oil (4 } \\
\mathrm{ml} / \mathrm{L})\end{array}$ & 1.75 & 1.69 & 1.81 & 1.58 & 1.23 & 2.00 & 2.12 & 1.92 \\
\hline $\begin{array}{l}\text { Dipping tubers in garlic oil (6 } \\
\mathrm{ml} / \mathrm{L})\end{array}$ & 1.75 & 1.69 & 1.52 & 1.45 & 1.61 & 1.59 & 1.83 & 1.80 \\
\hline $\begin{array}{l}\text { Dipping tubers in hydrogen } \\
\text { peroxide } 0.5 \%\end{array}$ & 1.75 & 1.69 & 1.49 & 1.58 & 1.64 & 1.76 & 1.88 & 1.96 \\
\hline $\begin{array}{l}\text { Dipping tubers in hydrogen } \\
\text { peroxide } 1 \%\end{array}$ & 1.75 & 1.69 & 1.75 & 1.73 & 1.48 & 1.83 & 2.07 & 2.29 \\
\hline $\begin{array}{l}\text { Dipping tubers in salicylic } \\
\text { acid } 800 \text { ppm }\end{array}$ & 1.75 & 1.69 & 1.95 & 1.26 & 1.75 & 1.63 & 2.17 & 2.09 \\
\hline $\begin{array}{l}\text { Dipping tubers in salicylic } \\
\text { acid 1000ppm }\end{array}$ & 1.75 & 1.69 & 1.46 & 1.19 & 1.89 & 1.47 & 1.78 & 1.94 \\
\hline \begin{tabular}{|l} 
Dipping tubers in \\
Paclopetrazol 250 ppm
\end{tabular} & 1.75 & 1.69 & 1.89 & 1.33 & 1.70 & 1.82 & 1.86 & 1.94 \\
\hline L.S.D. at $5 \%$ & NS & NS & 0.58 & 0.72 & .070 & 0.56 & 0.71 & 0.59 \\
\hline
\end{tabular}


Table (9): Effect of pre and post-harvest treatments on reducing sugar $(\mathrm{mg} / 100 \mathrm{~g})$ at the beginning of storage and at different period of storage 2, 4 and 6 months during 2007 and 2008.

\begin{tabular}{|c|c|c|c|c|c|c|c|c|}
\hline \multirow{4}{*}{ Treatments } & \multicolumn{8}{|c|}{ Reducing sugar } \\
\hline & \multirow{2}{*}{\multicolumn{2}{|c|}{0 time }} & \multicolumn{6}{|c|}{ Period in months } \\
\hline & & & \multicolumn{2}{|c|}{2} & \multicolumn{2}{|c|}{4} & \multicolumn{2}{|c|}{6} \\
\hline & 2007 & 2008 & 2007 & 2008 & 2007 & 2008 & 2007 & 2008 \\
\hline control & 0.87 & 0.97 & 0.88 & 1.15 & 1.72 & 1.72 & 1.87 & 2.05 \\
\hline $\begin{array}{l}\text { Spray plants with mint oil (4 } \\
\text { ml/L) }\end{array}$ & 0.71 & 1.08 & 1.11 & 1.17 & 1.76 & 1.82 & 2.06 & 2.26 \\
\hline $\begin{array}{l}\text { Spray plants with mint oil (6 } \\
\mathrm{ml} / \mathrm{L})\end{array}$ & 0.73 & 0.74 & 1.21 & 1.38 & 1.25 & 1.41 & 1.45 & 2.17 \\
\hline $\begin{array}{l}\text { Spray plants with caraway oil } \\
(4 \mathrm{ml} / \mathrm{L})\end{array}$ & 0.74 & 0.73 & 0.87 & 1.12 & 1.61 & 1.75 & 1.66 & 1.89 \\
\hline $\begin{array}{l}\text { Spray plants with caraway oil } \\
(6 \mathrm{ml} / \mathrm{L})\end{array}$ & 0.75 & 0.97 & 0.94 & 0.96 & 1.38 & 1.67 & 1.68 & 1.86 \\
\hline $\begin{array}{l}\text { Spray plants with garlic oil (4 } \\
\mathrm{ml} / \mathrm{L})\end{array}$ & 1.07 & 1.08 & 1.21 & 1.22 & 1.61 & 1.62 & 1.74 & 1.96 \\
\hline $\begin{array}{l}\text { Spray plants with garlic oil (6 } \\
\text { ml/L) }\end{array}$ & 1.19 & 1.25 & 1.32 & 1.50 & 1.71 & 1.91 & 1.97 & 2.01 \\
\hline $\begin{array}{l}\text { Dipping tubers in mint oil (4 } \\
\mathrm{ml} / \mathrm{L})\end{array}$ & 0.87 & 0.97 & 1.36 & 1.44 & 1.74 & 1.92 & 1.85 & 1.89 \\
\hline $\begin{array}{l}\text { Dipping tubers in mint oil (6 } \\
\mathrm{ml} / \mathrm{L})\end{array}$ & 0.87 & 0.97 & 1.38 & 1.45 & 1.98 & 1.88 & 1.81 & 2.05 \\
\hline $\begin{array}{l}\text { Dipping tubers in caraway oil } \\
(4 \mathrm{ml} / \mathrm{L})\end{array}$ & 0.87 & 0.97 & 1.47 & 1.53 & 1.83 & 1.80 & 1.88 & 1.93 \\
\hline $\begin{array}{l}\text { Dipping tubers in caraway oil } \\
(6 \mathrm{ml} / \mathrm{L})\end{array}$ & 0.87 & 0.97 & 1.21 & 1.41 & 1.74 & 1.89 & 1.82 & 1.94 \\
\hline $\begin{array}{l}\text { Dipping tubers in garlic oil (4 } \\
\text { ml/L) }\end{array}$ & 0.87 & 0.97 & 1.25 & 1.20 & 1.71 & 1.83 & 2.04 & 1.97 \\
\hline $\begin{array}{l}\text { Dipping tubers in garlic oil (6 } \\
\text { ml/L) }\end{array}$ & 0.87 & 0.97 & 0.88 & 0.97 & 1.05 & 1.23 & 1.75 & 1.78 \\
\hline $\begin{array}{l}\text { Dipping tubers in hydrogen } \\
\text { peroxide } 0.5 \%\end{array}$ & 0.87 & 0.97 & 0.94 & 0.93 & 1.36 & 1.32 & 1.54 & 1.63 \\
\hline $\begin{array}{l}\text { Dipping tubers in hydrogen } \\
\text { peroxide } 1 \%\end{array}$ & 0.87 & 0.97 & 0.96 & 0.98 & 1.19 & 1.08 & 1.71 & 1.74 \\
\hline $\begin{array}{l}\text { Dipping tubers in salicylic } \\
\text { acid } 800 \text { ppm }\end{array}$ & 0.87 & 0.97 & 1.03 & 1.20 & 1.17 & 1.10 & 1.37 & 1.49 \\
\hline $\begin{array}{l}\text { Dipping tubers in salicylic } \\
\text { acid 1000ppm }\end{array}$ & 0.87 & 0.97 & 0.92 & 1.15 & 1.23 & 0.90 & 1.14 & 1.36 \\
\hline \begin{tabular}{|l} 
Dipping tubers in \\
Paclopetrazol 250 ppm
\end{tabular} & 0.87 & 0.97 & 0.88 & 1.50 & 1.39 & 1.13 & 1.46 & 1.54 \\
\hline L.S.D. at $5 \%$ & Ns & Ns & 0.28 & 0.32 & 0.24 & 0.27 & 0.28 & 0.36 \\
\hline
\end{tabular}

\section{b-Total carbohydrate:-}

Table (10) shows decreased in total carbohydrate throughout the whole period of storage. Meanwhile, immersed of tuber in essential oil with higher concentration delayed decrease of total carbohydrate at the end of storage period. In contrary, control treatment which shows dramatically degradation and depletion in its tubers carbohydrate and other bioconstituent could be explained based on the harmful and degradable effects of high temperature oxidative stress. Such case might induce generation of elevated toxic level of oxygen radicals (Bowler et al., 1992 and Cakmac and Marschner, 1992 ; Rojas, et al., 2000) within stressed tissues. Thereby, degraded the cell membranes and allowed more $\mathrm{O} 2$ diffusion, raised respiration rate and sugars depletion (Daniels et al., 1996 and Vans and Hartman, 1987) in the same time Davies (1990), Trevanion and Kruger (1991) indicated that essential oils and/or their basic constituents slowing down the 
activity of carbohydrates and protein breakdown associated enzymatic systems as well as respiration and energy metabolism enzymes.

Understanding of ambient high temperature storage condition as a stressful factor, induce more serious internal oxidative stress, generation of elevated level of degradable and toxic reactive oxygen species (ROS) (O-2, $\mathrm{OH}-$ and H2O2) (Cakmak and Marschner, 1992 and Bowler et al., 1992; Jinxin et al., 1999 and Rojas et al., 2000). Probably as an actual factor beyond all potato storage problems (Rojas et al., 2000).

Finally, it could be concluded that dipping potato tuber twice with emulsion of garlic oil with $6 \mathrm{ml} / \mathrm{L}$ and salicylic acid $1000 \mathrm{ppm}$ were high effective on reduction of sprouting, weight loss and damage also two treatments were considered the best quality compare with other treatments.

Table (10): Effect of pre and post-harvest treatments on total carbohydrates (\%) of tubers at first storage and different period storage 2, 4 and 6 months during 2007 and 2008.

\begin{tabular}{|c|c|c|c|c|c|c|c|c|}
\hline \multirow{4}{*}{ Treatments } & \multicolumn{8}{|c|}{ Total carbohydrate (\%) } \\
\hline & \multirow{2}{*}{\multicolumn{2}{|c|}{0 time }} & \multicolumn{6}{|c|}{ Period in months } \\
\hline & & & \multicolumn{2}{|c|}{2} & \multicolumn{2}{|c|}{4} & \multicolumn{2}{|c|}{6} \\
\hline & 2007 & 2008 & 2007 & 2008 & 2007 & 2008 & 2007 & 2008 \\
\hline control & 24.80 & 23.17 & 21.18 & 19.63 & 20.98 & 19.19 & 18.08 & 18.75 \\
\hline $\begin{array}{l}\text { Spray plants with mint oil } \\
(4 \mathrm{ml} / \mathrm{L})\end{array}$ & 25.24 & 23.34 & 24.37 & 20.52 & 23.67 & 19.74 & 19.88 & 18.16 \\
\hline $\begin{array}{l}\text { Spray plants with mint oil } \\
(6 \mathrm{ml} / \mathrm{L})\end{array}$ & 23.35 & 23.57 & 22.55 & 21.56 & 20.91 & 18.18 & 17.67 & 16.37 \\
\hline $\begin{array}{l}\text { Spray plants with caraway } \\
\text { oil (4 ml/L) }\end{array}$ & 26.45 & 22.51 & 21.51 & 20.12 & 19.31 & 18.30 & 18.04 & 17.82 \\
\hline $\begin{array}{l}\text { Spray plants with caraway } \\
\text { oil }(6 \mathrm{ml} / \mathrm{L})\end{array}$ & 26.64 & 22.04 & 24.50 & 18.08 & 19.49 & 19.64 & 18.00 & 17.72 \\
\hline $\begin{array}{l}\text { Spray plants with garlic oil } \\
(4 \mathrm{ml} / \mathrm{L})\end{array}$ & 25.78 & 23.83 & 22.54 & 18.93 & 18.71 & 18.56 & 18.03 & 17.13 \\
\hline $\begin{array}{l}\text { Spray plants with garlic oil } \\
(6 \mathrm{ml} / \mathrm{L})\end{array}$ & 25.00 & 24.11 & 25.00 & 20.68 & 18.64 & 19.14 & 18.20 & 19.27 \\
\hline $\begin{array}{l}\text { Dipping tubers in mint oil } \\
(4 \mathrm{ml} / \mathrm{L})\end{array}$ & 24.80 & 23.17 & 21.88 & 21.40 & 21.03 & 18.16 & 18.47 & 19.67 \\
\hline $\begin{array}{l}\text { Dipping tubers in mint oil } \\
(6 \mathrm{ml} / \mathrm{L})\end{array}$ & 24.80 & 23.17 & 24.07 & 23.18 & 23.38 & 20.83 & 21.88 & 18.55 \\
\hline $\begin{array}{l}\text { Dipping tubers in caraway } \\
\text { oil (4 ml/L) }\end{array}$ & 24.80 & 23.17 & 24.35 & 20.67 & 23.39 & 20.46 & 18.77 & 18.16 \\
\hline $\begin{array}{l}\text { Dipping tubers in caraway } \\
\text { oil }(6 \mathrm{ml} / \mathrm{L})\end{array}$ & 24.80 & 23.17 & 23.08 & 21.62 & 22.69 & 20.63 & 22.12 & 20.08 \\
\hline $\begin{array}{l}\text { Dipping tubers in garlic oil } \\
(4 \mathrm{ml} / \mathrm{L})\end{array}$ & 24.80 & 23.17 & 24.03 & 20.17 & 20.47 & 20.61 & 18.14 & 19.23 \\
\hline $\begin{array}{l}\text { Dipping tubers in garlic oil } \\
(6 \mathrm{ml} / \mathrm{L})\end{array}$ & 24.80 & 23.17 & 24.14 & 22.58 & 22.82 & 21.19 & 21.11 & 19.27 \\
\hline $\begin{array}{l}\text { Dipping tubers in } \\
\text { hydrogen peroxide } 0.5 \%\end{array}$ & 24.80 & 23.17 & 24.14 & 22.36 & 22.22 & 19.96 & 20.79 & 18.94 \\
\hline $\begin{array}{l}\text { Dipping tubers in } \\
\text { hydrogen peroxide } 1 \%\end{array}$ & 24.80 & 23.17 & 21.54 & 21.00 & 20.62 & 20.43 & 20.74 & 18.37 \\
\hline $\begin{array}{l}\text { Dipping tubers in salicylic } \\
\text { acid } 800 \text { ppm }\end{array}$ & 24.80 & 23.17 & 24.18 & 19.06 & 21.71 & 18.81 & 19.02 & 18.03 \\
\hline $\begin{array}{l}\text { Dipping tubers in salicylic } \\
\text { acid 1000ppm }\end{array}$ & 24.80 & 23.17 & 23.71 & 21.10 & 22.58 & 19.82 & 21.54 & 19.33 \\
\hline $\begin{array}{l}\text { Dipping tubers in } \\
\text { Paclopetrazol } 250 \text { ppm }\end{array}$ & 24.80 & 23.17 & 23.87 & 19.59 & 22.06 & 20.02 & 20.56 & 19.17 \\
\hline L.S.D. at $5 \%$ & NS & NS & NS & Ns & NS & NS & 2.84 & 2.97 \\
\hline
\end{tabular}




\section{REFERENCES}

Barakat, M.A.S. (1996). Storability and productivity of Nili potato seed in relation to paclobutrazol (PP333). Menofiya J. Agric. Res., 21(1):135147.

Bowler, C.; M.V. Montogu and D. Inze (1992). Super oxide dismutase and stress tolerance. Ann. Rev. Plant Physiol. Plant Mol. Biol., 48:233-250

Cakmak, T. and H. Marschner. (1992): Magnesium deficiency and high light intensity enhance activities of super oxide dismutase, ascorbite peroxidase and glutathione rereductase in bean leaves. Plant Physiol .,98:1222-1227.

Coleman, W.; K.J. Greg (2001). Potato sprout growth suppression by menthone and neomenthol, volatile oil components of minthostachy, satureja, pystropogon and menthe species. Amer. J. Potatoes Res., 78:345-354.

Daniels, L.B.J. ; R.K. Prange ; W. Kalt ; G.L. Liew; J. Walsh; P. Dean and R. Coffin (1996). The effects of ozone and 1, 8-cineole on sprouting, fry color and sugars of stored Russet Burbank potatoes. Amer. Potato J., 73:469-481.

Davies, H.V. (1990). Carbohydrate metabolism during sprouting. Amer. Potato J., 67:815-827.

Dubois, M.; K.A. Gilles; J.K. Hamiltton; P.A. Rebers and F. Smith (1956). Colorimetic methods of determination of sugars and related substances. Anal. Chem., 28: 350-354.

El-Awady, A.E.A. (2002). Studies on potato storage. M.Sc. Thesis, Fac. of Agric., Mansoura Univ.

El-Awady, A.E.A. (2005). Studies on storing potato tubers out refrigerator using natural essential oils. Ph.D. Thesis, Fac. of Agric., Mansoura Univ.

Fathy, El-S.L.; A.M. Safa and A.E. Amal (2005). Using of natural antioxidants for improving storability of potatoes. The 6th Arabian Conference for Horticulture, Ismailia, Egypt.

Fathy, El-S.L.; Z.M. Khder and E.M. Al-Said (2003). New techniques for effective and safe storing of potatoes at the ambient temperature. J. Agric. Sci. Mansoura Univ., 28(6):4959-4973.

Hartmans, K.J.; P. Diepenhorst; W. Bakker and L.G.M. Gorris (1995). The use of carvone in agriculture: Sprout suppression of potatoes and antifungal activity against potato tuber and other plant diseases. Industrial Crop and Products, 4:3-13.

Jinxin, Y.I.; C. Jinghua and Y. Qiying (1999). Preliminary study on methods for identification of tolerance to low temperature. Jiangsu Agric. Sci., 5:51-53.

Knobloch, K. ; H. Weigand; N. Weis; H.M. Schwarm and H. Vigenschow (1986). Action of terpenoids on energy metabolism. In: E.J. Brunke (Editor), Progress in Essential Oil Research Walter de Gruyter, New York, PP. 429-445. 
Naguib, M. I. (1964). Effect of sevin on the carbohydrate and nitrogen metabolism during germination of cotton seeds. Indian J. Exp. Biol. 2:149.

Nelson, N. (1944). A photometric adaptation of the somogyi method for the determination of glucose. J. Biol. Chem. 153:375-380.

Oosterhaven, K. ; K.J. Hartmans and H.J. Huizing (1993). Inhibition of potato (Solanum tuberosum) sprout growth by the monoterpene s-carvone: reduction of 3-hydroxy-3-methylglutaryl coenzyme A reductase activity without effect on its mRNA level. J. Plant Physiol., 141:463-469 .

Rabbany, A.B.M. and F. Mizutani (1996). Effect of essential oils on ethylene production and ACC content in apple fruit and peach seed tissue. J. Japanese Soc. Hort. Sci., 65(1):7-13.

Ranganna, S. (1979). Manual of Analysis of Fruit and Vegetable Products. TATA McGrow Publishing Company Limited. New Delhi, PP. 634.

Rojas, J.A.; F. Dejaegher; M. Abdalla-Kotb and D.V. Jardin (2000). Expression and activity of antioxidant enzymes during potato tuber dormancy. Potato Res., 43:383-393.

SAS (2005). User's Guide statistics, version 5 Ed. SAS Institute, Inc, Cary, NC, USA.

Trevanion, S.J. and N.J. Kruger (1991). Effect of temperature on the kinetic properties of pyrophosphate: fructose 6-phosphate phosphotransferase from potato tuber. J. Plant Physiol., 137:753-759.

Van der Plas. L.H.W., (1987). potato tuber storage: biochemical and physiological changes. In: Bajaj, Y.P.S. (Editor)Biotechnology in Agriculture and Forestry. Vol. 3: potato, springerverlag Berlin Heidelberg. pp.190-135.

Vanes, A. and K.J. Hartmans (1987). Respiration. PP. 133-140, In: Rastovski, A. Vanes, et al., Storage of potatoes, Post-harvest, store design, storage practice, handling. Pudoc., Wageningen.

Vaughn, S.F. and G.F. Spencer (1991). Volatile monoterpenes inhibit potato tuber sprouting. Amer. Potato J., 68:821-830.

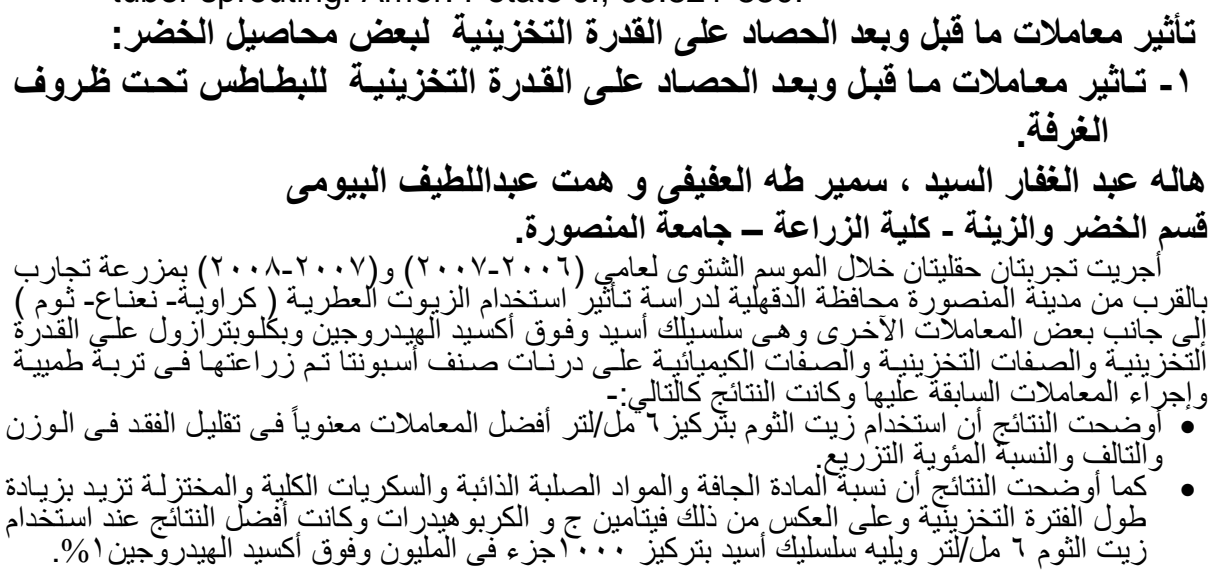


E EL-Sayed, Hala A. et al. 